University of Nebraska - Lincoln

DigitalCommons@University of Nebraska - Lincoln

$11-1-1999$

\title{
Imaging point defects in a liquid environment: A model AFM study
}

\author{
Kenichiro Koga \\ University of Nebraska-Lincoln, koga@cc.okayama-u.ac.jp \\ Xiao Cheng Zeng \\ University of Nebraska-Lincoln, xzeng1@unl.edu
}

Follow this and additional works at: https://digitalcommons.unl.edu/chemzeng

Part of the Chemistry Commons

Koga, Kenichiro and Zeng, Xiao Cheng, "Imaging point defects in a liquid environment: A model AFM study" (1999). Xiao Cheng Zeng Publications. 56.

https://digitalcommons.unl.edu/chemzeng/56

This Article is brought to you for free and open access by the Published Research - Department of Chemistry at DigitalCommons@University of Nebraska - Lincoln. It has been accepted for inclusion in Xiao Cheng Zeng Publications by an authorized administrator of DigitalCommons@University of Nebraska - Lincoln. 


\title{
Imaging point defects in a liquid environment: A model AFM study
}

\author{
Kenichiro Koga* and X. C. Zeng \\ Chemistry Department and Center for Materials Research and Analysis, University of Nebraska-Lincoln, Lincoln, Nebraska 68588
}

(Received 23 March 1999)

\begin{abstract}
Effects of the liquid on atomic force microscopy (AFM) imaging are examined for a model system consisting of a hexagonal flake of seven Lennard-Jones (LJ) atoms as a multiatom tip, a monolayer of LJ crystal containing a single point defect as a substrate, and three-site model water as the liquid. A previous simulation [Koutsos et al., Europhys. Lett. 26, 103 (1994)] has shown that the true atomic resolution of a point defect cannot be achieved in vacuum by use of the multiatom tip. Here we examine the feasibility of such atomic resolution when both the tip and substrate are immersed in a liquid. The liquid-induced interaction between the tip and the substrate is evaluated in the framework of the integral equation theory of molecular fluids extended for the system consisting of an infinitely large crystal. It is found that the potential of mean force indeed manifests the point defect even when the tip and the substrate are a few Angstroms apart. This implies that in the liquid environment the point defect could be discerned in the constant-height mode of AFM measurement. For the constant-load mode of AFM, a characteristic potential can be used to determine the stable, metastable, and unstable vertical position (height) of the tip at any lateral position. The contour surface of the stable heights reveals periodic features of the underlying lattice of the substrate except in the vicinity of the defect, provided certain load is applied. [S0163-1829(99)10543-5]
\end{abstract}

\section{INTRODUCTION}

Atomic force microscopy (AFM) aims to "see" atomic details of surfaces by detecting the force acting on the AFM tip. ${ }^{1}$ In vacuum the force acting on the AFM tip is essentially due to the intermolecular interactions between atoms of the tip and those of the surface. Therefore, the force curve-the force in the direction vertical to the surface versus the tipsurface distance-reflects essentially the same feature as that of the intermolecular force, that is, a short-range strong repulsion and a long-range weak attraction.

When both the tip and surface are immersed in a liquid, the liquid-mediated force arises in addition to the direct tipsurface interaction. ${ }^{2-4}$ Thus, the potential of mean force of the tip surface is expressed as

$$
W(\mathbf{R})=U(\mathbf{R})+\delta A(\mathbf{R})
$$

where $U$ is the potential due to the direct interaction between the tip and surface; $\delta A$ is the liquid-mediated potential. Both potentials are a function of the position vector $\mathbf{R}$ of the tip. (Let the unit vector $\mathbf{e}_{z}$ denote the direction vertical to the surface, and $\mathbf{e}_{x}$ and $\mathbf{e}_{y}$ the directions parallel to the surface.) The liquid-mediated potential $\delta A$, when plotted against $R_{z}$, has an oscillatory character due to the layering structure of liquid near the surface.

In the constant-height scanning mode of AFM, the force $\mathbf{F}(=-\nabla W)$ acting on the tip is measured and recorded to construct the image of the surface. In the constant-load mode, on the other hand, the position $\mathbf{R}$ of the tip is measured in general; in an ideal situation where one can precisely control the lateral position $\left(R_{x}, R_{y}\right)$ of the tip, "the height of the tip" $R_{z}$ is measured under a constant load $F_{z}^{\text {ext }}$. In the latter case, one can define a function based on the potential of mean force,

$$
\Psi=W-F_{z}^{\mathrm{ext}} R_{z},
$$

which serves as a new potential analog to the thermodynamic potential. As a result, the equilibrium height of the tip $R_{z}^{\text {eq }}$ can be determined by the condition

$$
\delta \Psi=0
$$

where the variation is taken under the condition of fixed $R_{x}$, $R_{y}, F_{z}^{\text {ext }}$, and temperature $T$. Equation (3) is equivalent to the force balance equation $F_{z}+F_{z}^{\text {ext }}=0$, where $F_{z}$ is the $z$ component of $\mathbf{F}$. In general, the potential $\Psi$ may have more than one local minimum and maximum depending on the load as illustrated in Fig. 1. This contrasts with the characteristic potential in vacuum, which generally has only one minimum and no maximum irrespective of the magnitude of the load.

In the previous paper ${ }^{5}$ we showed that the oscillatory behavior of $\Psi$ can result in discontinuous scanning trajectories for a single-atom tip, even if the surface is defect free. AFM experiments in the liquid environment have also shown that the force acting on the tip can be oscillatory when the tip approaches the surface vertically, reflecting the layering structure of liquid molecules near the surface. ${ }^{6-8}$

In this paper we extend our previous theoretical study to examine effects of the liquid on the AFM image of a surface containing a point defect or specifically a vacancy. Unlike the previous study, here a multiatom tip is considered. Such a multiatom tip and surface system have been studied from a computer simulation, but in a vacuum environment. ${ }^{9}$ That study shows that the multiatom tip cannot detect a vacancy. In other words, true atomic resolution cannot be achieved with the multiatom tip in the vacuum environment. When the tip scans the surface containing a vacancy, the AFM image is deceptive because it only reveals the structure of the multiatom tip but not the vacancy. The difficulty in achieving true 


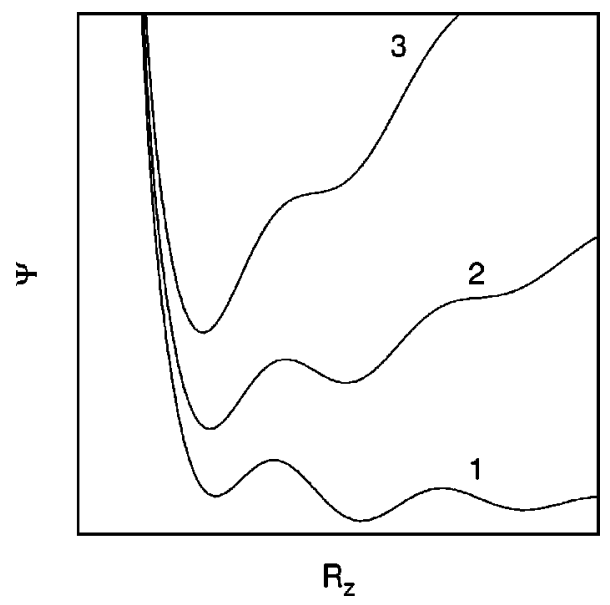

FIG. 1. Schematic $\Psi-R_{z}$ diagram for three different loads: curve 1 (low load), curve 2 (medium load), and curve 3 (high load). Curve 1 shows that at a low load the second minimum can be the global minimum whereas the first minimum is a local minimum corresponding to a metastable state. As the load increases, the first minimum turns to be the global minimum (curve 2) and eventually will be the only minimum (curve 3 ) at a sufficiently high load.

atomic resolution of this kind of defect in the vacuum environment can be understood from the fact that the total force acting on the tip is approximately sum of the short-ranged pairwise repulsive interactions between atoms of the tip and those of the surface. On the other hand, in a liquid environment the liquid-mediated interaction between the tip and surface is long-ranged, oscillatory, and nonpairwise additive. Thus when the $\delta A$ term in Eq. (1) is comparable to the $U$ term or dominant, the AFM image should be qualitatively different from that in vacuum; however, it is still unclear whether a liquid environment is helpful or not to achieve true atomic resolution. The purpose of this work is to shed some light on this issue via a simple AFM model study.

The liquid-induced (also called solvent-induced) interaction is calculated from the supermolecule approach ${ }^{10,11}$ based on the reference interaction site model (RISM) integral equation theory. ${ }^{12}$ This approach was originally developed for homogeneous systems. Recently this approach was extended by us to treat inhomogeneous systems ${ }^{13,5}$ using the polymer RISM equation. ${ }^{14}$ This extension enables us to calculate the solvent-mediated interactions for various systems, such as those between two solute molecules near a structured wall, between a solute molecule and the wall, and between two structured walls. It turns out that for our purpose this approach is more effective than the computer simulation.

In this study, the models of the AFM tip and the surface are treated to be rigid so that neither vibrational motions nor deformation will be considered. We note that there are several studies involving more realistic models of the AFM tip and surface in vacuum. ${ }^{15}$ Here we adopt the simple rigid model for tip and surface mainly because the focus is to show effects of the liquid (i.e., solvent) on the AFM images.

In the following section we illustrate the model system and overview the methodology for the calculation of the solvent-induced contribution to the total potential. In Sec. III we present results of numerical calculation and possible implication of these results on the AFM measurements. Concluding remarks are given in Sec. IV.

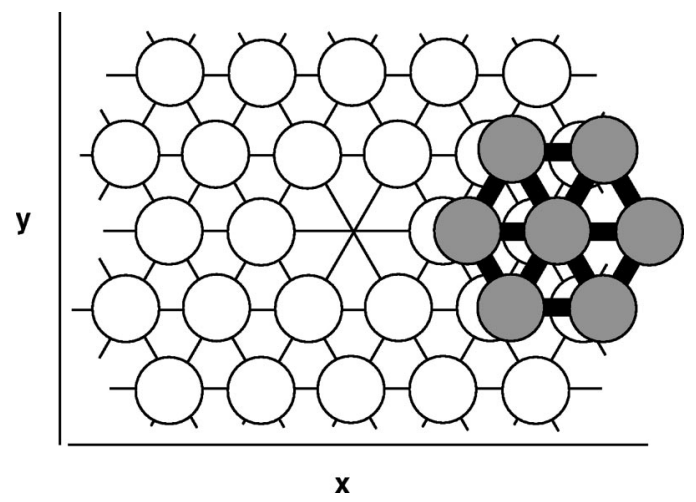

FIG. 2. Schematic diagram of the hexagonal-close-packed substrate (white-colored atoms) and the hexagonal disklike tip (seven grey-colored atoms with thick bonds). The origin of the coordinates is set at the lattice site of the vacancy. The $z$ axis is perpendicular to the substrate.

\section{MODELS AND METHOD}

\section{A. Models of AFM tip, substrate, and solvent}

We consider the system consisting of an AFM tip, a substrate, and a pure solvent: the tip is taken to be a rigid hexagonal flake consisting of $N_{t}(=7)$ atoms possessing a hexagonal-close-packed (hcp) lattice structure; the substrate is a rigid monolayer of $N_{s}$ atoms also having the hep lattice structure (with the same lattice constant as that of the tip) containing a single vacancy at a lattice site (Fig. 2). Both the tip and the substrate are immersed in liquid water of the constant number density $\rho$ and temperature $T$.

The orientation of the multiatom tip is fixed such that the plane of the tip is always parallel to the substrate and the in-plane orientation of the tip coincides with that of the substrate. The origin of the Cartesian coordinate is placed at the lattice site of the atomic hole, the $z$ axis is set perpendicular to the substrate, and the $x$ axis parallel to one of the unit vectors of the hexagonal lattice of the substrate. The position of the tip is specified by its center-of-mass coordinate.

Two types of ideal scanning modes are considered: the constant-height mode in which $R_{z}$ is kept constant and the constant-load mode in which $F_{z}^{\text {ext }}{ }^{2}$ is kept constant; in either mode $R_{x}$ and $R_{y}$ are controlled precisely during the scan.

The tip-substrate direct interaction is given by a pairwise sum $U(\mathbf{R})=\Sigma_{i=1}^{N_{t}} \Sigma_{j=1}^{N_{s}} \phi^{\mathrm{LJ}}\left(r_{i j}\right)$, where $\phi^{\mathrm{LJ}}$ is the LennardJones (LJ) potential: $\phi^{\mathrm{LJ}}(r)=4 \epsilon_{s}\left[\left(\sigma_{s} / r\right)^{12}-\left(\sigma_{s} / r\right)^{6}\right]$ and $r_{i j}$ denotes the distance between $i$ th atom of the tip and $j$ th atom of the substrate; $\epsilon_{s}$ and $\sigma_{s}$ are the LJ energy and size parameters. We use the parameters for a ten-site model of naphthalene: ${ }^{16} \epsilon_{s} / k=67.1 \mathrm{~K}$ and $\sigma_{s}=3.395 \AA$, where $k$ denotes the Boltzmann constant. The molecular potential of water is described by a slightly modified TIPS site-site interaction potential. ${ }^{17}$ The water-tip and water-substrate intermolecular interactions are described by LJ potentials with the parameters determined by combining rules, $\epsilon_{w s}=\sqrt{\epsilon_{w} \epsilon_{s}}$ and $\sigma_{w s}=\left(\sigma_{w}+\sigma_{s}\right) / 2$, where $\epsilon_{w}$ and $\sigma_{w}$ are the LJ energy and size parameters of the oxygen-oxygen interaction in the TIPS model. The lattice constant $l_{a}$ of the monolayer substrate is set to be $2^{1 / 6} \sigma_{s} \sim 3.81 \AA$. Temperature is set at $298.15 \mathrm{~K}$ and the number density of water $\rho$ at $1 \mathrm{~g} / \mathrm{cm}^{3}$. 


\section{B. Quasistatic scanning process}

In a typical AFM experiment, the time scale for the tip to scan over one nanometer is of the order of one millisecond, much longer than the time scale required for the attainment of thermodynamic equilibrium of a liquid. Thus, it is reasonable to assume that the scanning process is quasistatic, that is, a succession of equilibrium states each of which is completely described by a set of parameters suitable for a given experimental condition. In our calculation each state is specified by $\rho, T, \mathbf{R}$ under the constant-height mode and by $\rho, T$, $R_{x}, R_{y}$, and $F_{z}^{\text {ext }}$ under the constant-load mode. During the scanning process, the density and temperature of the liquid are kept constant. Since the system is taken to be infinitely large in the calculation described below, other thermodynamic properties such as the chemical potential and the pressure of the liquid would also be constant during the scanning process.

\section{Calculation of solvent-induced interaction}

The solvent-induced interaction $\delta A(\mathbf{R})$ is calculated from the supermolecule approach ${ }^{10,11}$ based on the RISM integral equation theory. ${ }^{12}$ In this approach a pair of solute molecules is considered as a supermolecule with a fixed relative configuration $\mathbf{R}$ dissolved in the solvent. (In the present case the tip and the substrate are two solute molecules in the liquid.) First, one calculates the solvation free energy ${ }^{18}$ of the supermolecule as a function of $\mathbf{R}$, which is defined as

$$
\Delta A^{*}(\mathbf{R})=-k T \ln Z_{N}^{S}(\mathbf{R}) / Z_{N}^{0},
$$

where $Z_{N}^{s}(\mathbf{R})=\int d \mathbf{r}^{N} \exp \left[-V\left(\mathbf{r}^{N} ; \mathbf{R}\right) / k T\right]$ is the configuration integral of the system of $N$ solvent molecules and the supermolecule placed at a fixed position in a space whereas $Z_{N}^{0}$ $=\int d \mathbf{r}^{N} \exp \left[-V^{0}\left(\mathbf{r}^{N}\right) / k T\right]$ is the configuration integral of the system without the supermolecule. Next, one calculates the potential $\delta A(\mathbf{R})$ due to the solvent-mediated interaction, which is the solvation free energy $\Delta A^{*}(\mathbf{R})$ relative to an arbitrary standard. Taking the standard to be $\Delta A_{\infty}^{*}$, the solvation free energy at $R_{z}=\infty, \delta A(\mathbf{R})$ is written as

$$
\delta A(\mathbf{R})=\Delta A^{*}(\mathbf{R})-\Delta A_{\infty}^{*}
$$

In the framework of the extended RISM theory, ${ }^{19}$ the solvation free energy of a solute molecule at infinite dilution is given by ${ }^{20,21}$

$$
\Delta A^{*}=\rho k T \sum_{i j} \int d \mathbf{r}\left\{\frac{1}{2}\left[h_{i j}(r)\right]^{2}-c_{i j}(r)-\frac{1}{2} h_{i j}(r) c_{i j}(r)\right\},
$$

where $h_{i j}$ and $c_{i j}$ denote, respectively, the correlation function and the direct correlation function between site $i$ of a solvent molecule and site $j$ of a solute molecule. The correlation functions are obtained by solving the RISM integral equation numerically. No further approximation to the original RISM theory is involved in the supermolecule approach. Previous studies using the supermolecule approach have shown semiquantitative agreements with computer simulation and experiments for nonpolar, ${ }^{21}$ polar, ${ }^{11}$ and ionic ${ }^{10}$ solutes in solvents.
Implementation of the supermolecule approach to $\delta A(\mathbf{R})$ for the AFM tip-substrate system is summarized as follows.

(i) Two-dimensional periodicity in the $x$ and $y$ directions is introduced to the supermolecule consisting of the monolayer substrate and the AFM tip. Specifically, the periodic unit consists of the tip $\left(N_{t}=7\right)$ and a rectangular section of the substrate $\left(N_{s}=11 \times 12-1=131\right)$ with side length $L_{x}$ $=11 l_{a}$ and $L_{y}=6 \sqrt{3} l_{a}$. The periodic boundary condition is essential in order to avoid any edge effect of the substrate on the solvent-mediated interaction.

(ii) Long-ranged intramolecular correlations in the supermolecule are truncated smoothly. Specifically, $\hat{\mathbf{w}}$ the intramolecular site-site correlation matrix in the reciprocal space $^{22}$ is modified by multiplying each matrix element $\hat{w}_{i j}$ by a switching function $f\left(r_{i j}\right)$ defined as 1 if $r_{i j}<r_{t}$, $\frac{1}{2}\left\{\cos \left[\pi\left(r_{i j}-r_{t}\right) /\left(r_{c}-r_{t}\right)\right]+1\right\}$ if $r_{t}<r_{i j}<r_{c}$, and 0 if $r_{c}<r_{i j}$, where $r_{t}=4.5 l_{a}, r_{c}=5.5 l_{a}$, and $r_{i j}$ is the distance between atoms $i$ and $j$ in the periodic system (where the minimum image convention is applied). This procedure ensures that the partial sum of infinite elements $\hat{w}_{i j}$ converges. Note that no correlation between the tip and any image tip in the surrounding unit cells is included because the shortest site-site distance between the tips is larger than $r_{c}$.

(iii) The correlation functions $h_{i j}$ and $c_{i j}$ between the interaction sites of the water molecule and those in the unit cell of the supermolecule are numerically calculated by the standard iteration scheme. The solvation free energy $A *$ of the supermolecule with a fixed tip-substrate relative configuration $\mathbf{R}$ is then obtained from Eq. (5). Note that the correlation functions associated with the interaction sites of the substrate beyond the unit cell of the supermolecule are excluded in the calculation of $\Delta A^{*}$. This is justified by the fact that the unit cell is chosen sufficiently large so that the excluded correlation functions have no significant contribution to the solventinduced interaction $\delta A$.

(iv) The solvent-induced interaction $\delta A$ is calculated at $33 \times 9 \times 10$ grid points covering a rectangular region: 0 $\leqslant R_{x} \leqslant 4.125 l_{a}, \quad 0 \leqslant R_{y} \leqslant \sqrt{3} l_{a}$, and $0.7 \sigma_{w s} \leqslant R_{z} \leqslant 2 \sigma_{w s}$. Values of $\delta A$ at finer grid points are then evaluated using an interpolation method.

\section{RESULTS AND DISCUSSION}

\section{A. Constant-height mode}

Figure 3 shows the numerical results of $W$ and $U$ for a scan over the defect along the path $R_{y}=0$ under the constant-height mode. Three different values of height $\left(R_{z}\right)$ are examined. The structure of the surface manifests itself in $U$ only when $R_{z}$ is close to or smaller than $\sigma_{w s}$ (the thin curve marked 1); however, the surface structure does manifest itself in $W$ even when $R_{z}$ is larger than $\sigma_{w s}$, at which the tip never touches the surface (the thick curves marked 2 and 3).

The potential-energy surfaces at the constant-height mode, defined by $W\left(R_{x}, R_{y} ; R_{z}\right)$ with $R_{z}$ being kept constant, are plotted in Fig. 4 for the three different values of $R_{z}$. When the tip-surface distance $R_{z}$ is close to the repulsive diameter $\sigma_{w s}\left(R_{z}=3.02 \AA\right)$, one can clearly see in $W$ the periodic lattice structure of the substrate. However, it is difficult to see the location of the point defect since a pro- 

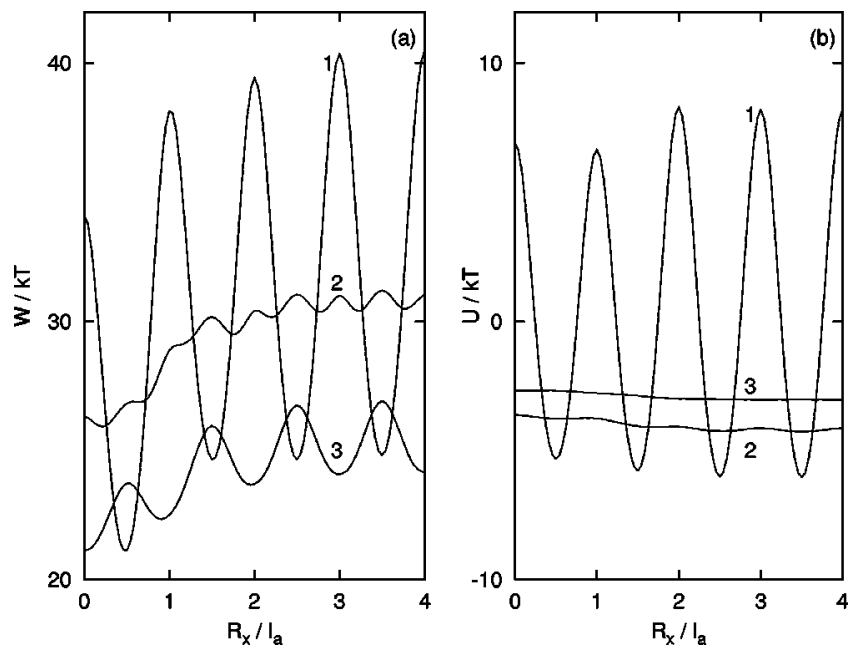

FIG. 3. Variations of $W$ (a) and $U$ (b) in units of $k T$ in the constant-height scanning mode. The abscissa is the $x$ coordinate of the tip $R_{x}$ in units of $l_{a}$; the $y$ coordinate $R_{y}$ is kept constant at 0 $\AA$. The curves marked 1,2 , and 3 correspond to the three tip heights $R_{z}=3.02 \AA, 3.92 \AA$, and $4.36 \AA$, respectively. Note that the single-point defect (a vacancy) is located at the origin of the coordinates.
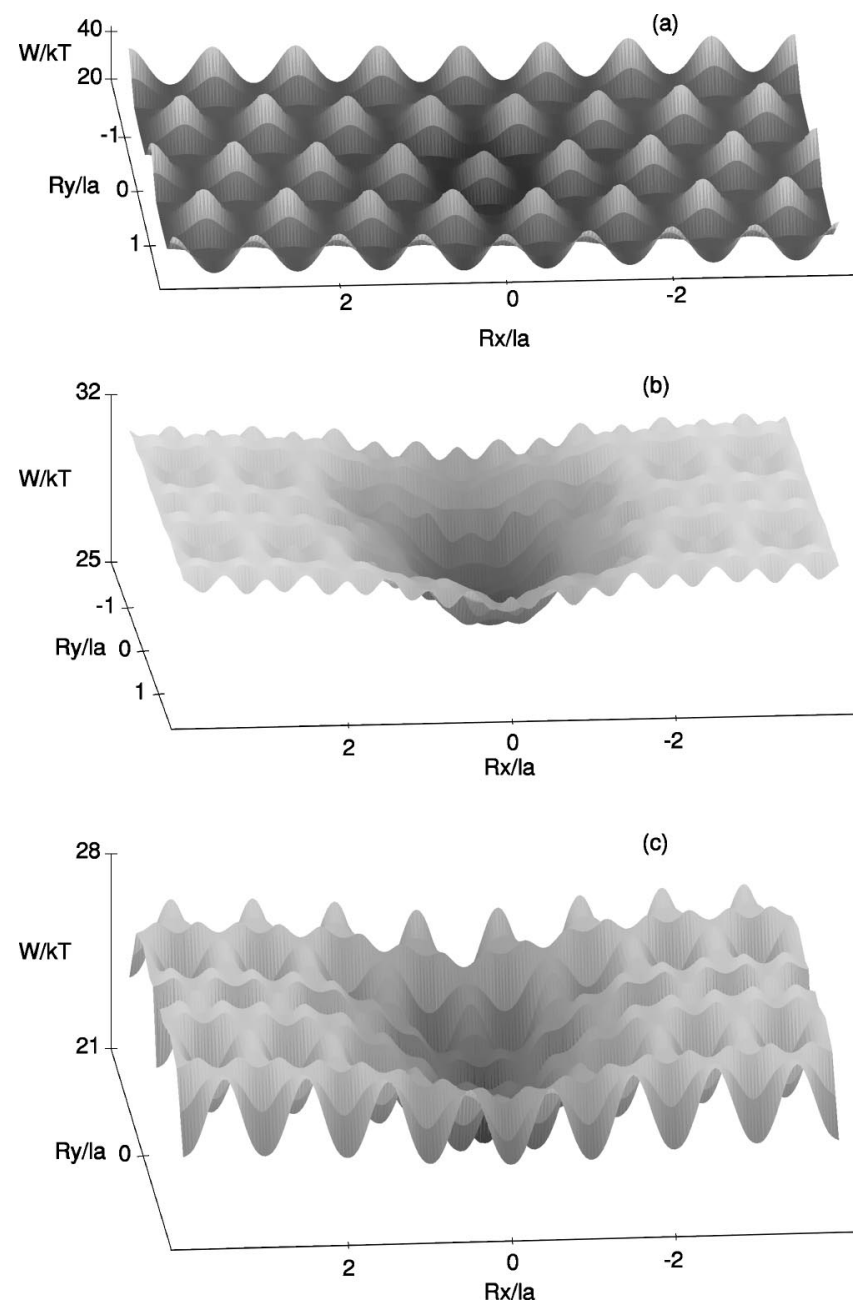

FIG. 4. Potential- (of mean force) energy surface $W / k T$ for the three constant heights: $R_{z}=3.02 \AA$ (a), $3.92 \AA$ (b), and $4.36 \AA$ (c). nounced peak similar to other peaks is found at the place of the atomic hole. This is because at the short distance the direct tip-surface potential $U$ is dominant in $W$, compared to $\delta A$. The corresponding potential-energy surface in vacuum defined by $U$ instead of $W$ is more or less the same at this height due to the same reason.

As the AFM tip is moved away from the surface, the strong direct interaction diminishes considerably, and $\delta A$, the solvent-mediated interaction, becomes the main contribution to $W$ because $\delta A$ is a long-ranged interaction due to the packing effect of solvent molecules near the surface. Shown in Figs. 4(b) and 4(c) are examples of the potential-energy surface in which $\delta A$ is dominant. When $R_{z}=3.92 \AA$ [Fig. $4(\mathrm{~b})]$, one can observe clearly a large minimum in $W$ at the location of the point defect. Overall, the $W$ surface is smooth except near the defect where it shows a minimum. However, the lattice structure is unclear at this height. At a larger height $R_{z}=4.36 \AA$ [Fig. 4(c)], $W$ becomes rugged again reflecting the structure of the substrate. Moreover, the lowest potential well is located at the position of the point defect.

\section{B. Constant-load mode}

In the constant-load mode, Eq. (3) gives values of height $\left(R_{z}\right)$ at which the average force $F_{z}$ balances with the external force $F_{z}^{\text {ext }}$. In general, several values of $R_{z}$ are found to satisfy Eq. (3) at fixed $R_{x}$ and $R_{y}$, due to the layering structure of the liquid (see Fig. 1). These values can be classified as follows: the stable height at which $\Psi$ is the global minimum, the metastable heights at which $\delta^{2} \Psi>0$ but $\Psi$ is not the global minimum, and the unstable heights at which $\delta^{2} \Psi<0$, where the variations are taken under the same condition as Eq. (3). The stable trajectory is then defined as a series of the stable heights for a given scanning path $\left[R_{y}\right.$ $\left.=f\left(R_{x}\right)\right]$.

Figure 5 shows the numerical results of the trajectories [sets of heights that satisfy Eq. (3)] along the path $R_{y}=0$ for three different values of load $F_{z}^{\text {ext }}:-0.2 \mathrm{nN},-0.4 \mathrm{nN}$, and $-0.6 \mathrm{nN}$. (These loads are comparable to typical load per atom in laboratory AFM experiments.) One finds three continuous series of heights, marked 1, 2, and 3, in Figs. 5(a) and 5(b) and one continuous series of heights in Figs. 5(c). Series 1 corresponds to a trajectory of the tip being always in contact with the substrate $\left(R_{z} \sim 3 \AA\right)$ whereas series 3 corresponds to a trajectory of the tip being separated from the substrate by one hydration layer $\left(R_{z} \sim 5 \AA\right)$. Series 2 is a set of the unstable heights that separates the stable and metastable heights. One may consider Fig. 5 as a map of the potential surface $\Psi\left(R_{x}, R_{z}\right)$ at fixed $R_{y}$; then series 1 and series 3 are valleys of the potential surface whereas series 2 is a ridge of the potential barrier.

The stable trajectory (denoted by dots) belongs to either series 1 or series 3 depending on $R_{x}$ and $F_{z}^{\text {ext }}$. For $F_{z}^{\text {ext }}=$ $-0.2 \mathrm{nN}$ [Fig. 5(a)], series 3 is the most stable trajectory when $n-0.3<R_{x} / l_{a}<n+0.3$ with $n$ an arbitrary integer; otherwise series 1 is the most stable. Consequently, the stable trajectory of the AFM tip is discontinuous as previously found for a single-atom tip scanning over a substrate containing no defect. ${ }^{5}$ For $F_{z}^{\text {ext }}=-0.4 \mathrm{nN}$ [Fig. 5(b)], series 1 is the most stable when $n-0.1<R_{x}<n+0.1$ except $n=0$, otherwise series 3 is the most stable. Thus, the stable 

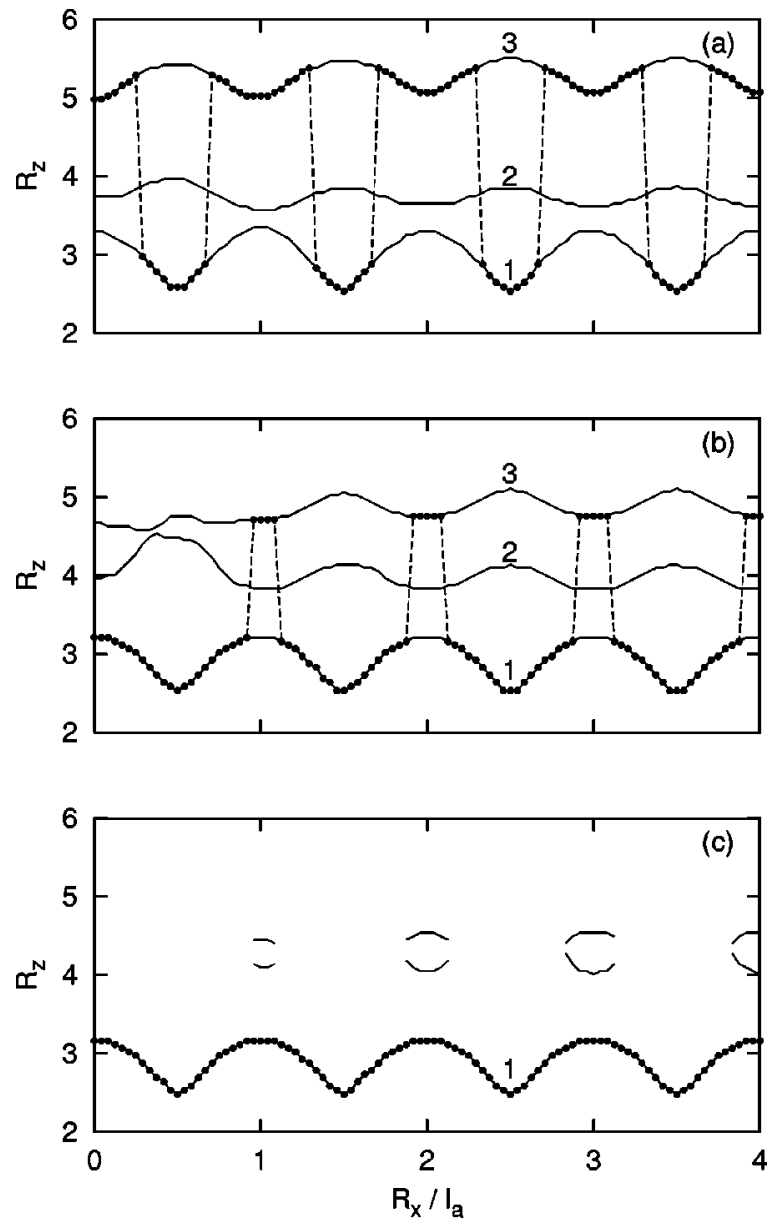

FIG. 5. Heights $R_{z}$ of the tip [which satisfy Eq. (3)] in the constant-load scanning processes along the path $R_{y}=0: F_{z}^{\mathrm{ext}}=$ $-0.2 \mathrm{nN}$ (a), $-0.4 \mathrm{nN}$ (b), and $-0.6 \mathrm{nN}$ (c). $R_{z}$ is in units of $\AA$. Curves marked 1 and 3 correspond to stable or metastable height whereas curves marked 2 correspond to unstable height. The most stable heights for each load are denoted by black dots.

trajectory has a discontinuous feature except around $R_{x} / l_{a}$ $=0$. The missing of the discontinuous jump from series 3 to series 1 around $R_{x} / l_{a}=0$ reflects the existence of the singlepoint defect (an atomic hole) in the substrate. Finally, for $F_{z}^{\mathrm{ext}}=-0.6 \mathrm{nN}[$ Fig. 5(c)], which is the highest load among the three, one finds that series 1 is the most stable in the entire range of $R_{x}$; the stable trajectory is therefore continuous as in vacuum environment.

Whether the tip follows the stable trajectory or jumps between the stable and the metastable states depends on the potential-energy barrier height between two states. The potential-energy barrier height is defined as the difference in $\Psi$ between the most stable height and the unstable height at given $R_{x}$ and $R_{y}$. We find that the potential barrier height ranges from $5 k T$ to $20 k T$ at $F_{z}^{\text {ext }}=-0.2 \mathrm{nN}$ [Fig. 5(a)] and from $3 k T$ to $25 k T$ at $F_{z}^{\text {ext }}=-0.4 \mathrm{nN}$ [Fig. 5(b)]. These values are comparable to the thermal energy. Thus, it is unlikely that the AFM tip follows only the most stable heights upon scanning the substrate in the liquid environment; rather, the tip may jump between stable and metastable heights. Similar conclusions have also been made from a laboratory experiment. ${ }^{7}$ Our calculation indicates that the trajectory of

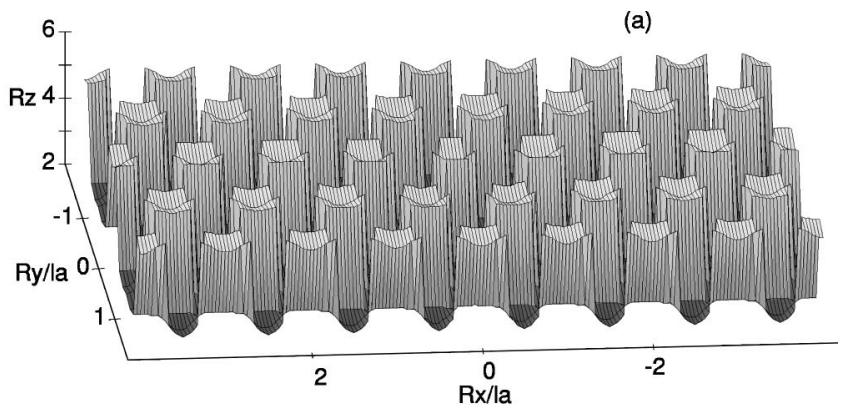

(b)

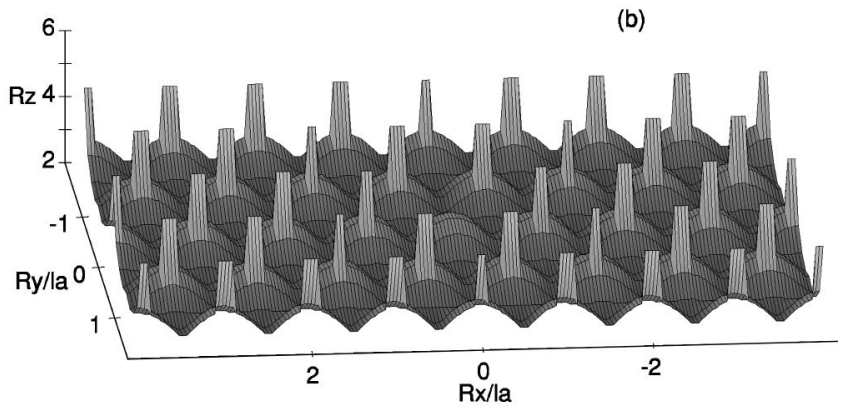

(c)

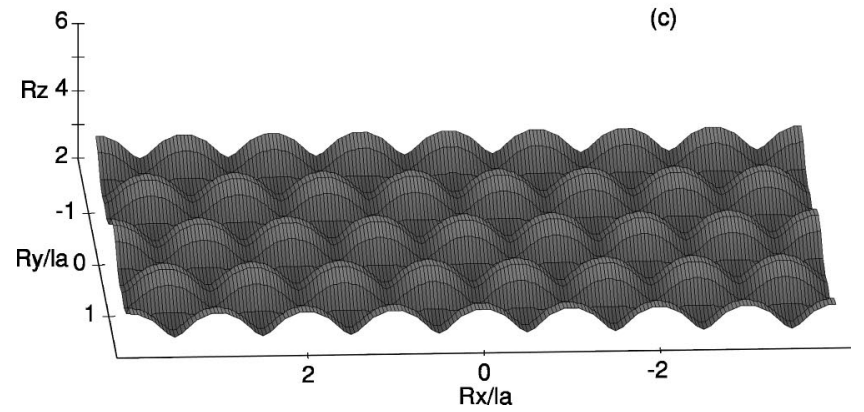

FIG. 6. The most probable height $R_{z}$ as a function of the lateral position $\left(R_{x}, R_{y}\right)$ of the tip under the constant load conditions: $F_{z}^{\mathrm{ext}}=-0.2 \mathrm{nN}(\mathrm{a}),-0.4 \mathrm{nN}(\mathrm{b})$, and $-0.6 \mathrm{nN}(\mathrm{c}) . R_{z}$ is in units of A.

the AFM tip in liquid cannot be pinned down uniquely; however, it can be defined as a series of the most probable heights.

It is interesting to see the most probable height $R_{z}$ of the AFM tip as a function of $R_{x}$ and $R_{y}$. Plotted in Fig. 6 are the numerical results of $R_{z}$ under the same three constant loads as shown in Fig. 5. Under the condition $F_{z}^{\text {ext }}=-0.2 \mathrm{nN}, R_{z}$ is periodic and discontinuous over the substrate including the position of the single-point defect. The existence of the defect is hardly seen from this result. However, for $F_{z}^{\text {ext }}=$ $-0.4 \mathrm{nN}, R_{z}$ is continuous in a region around the defect; otherwise it exhibits periodic and discontinuous feature. Under a higher load, $F_{z}^{\text {ext }}=-0.6 \mathrm{nN}$, the discontinuous feature disappears in $R_{z}$, indicating that the tip is always in contact with the substrate. In this case one can hardly discern the single-point defect because the tip is not "sharp" compared to the single-atom one.

\section{CONCLUDING REMARKS}

In this study, effects of the liquid on the total potential of a multiatom AFM tip as well as on the tip's trajectory when scanning over a substrate containing a single-point defect are 
examined within the framework of the RISM integral equation theory. At the constant-height mode the potential of mean force reflects the existence of the defect when the tip and the substrate are close to but not in contact with each other. In this case the friction force in the vicinity of the vacancy will become larger in liquid than in vacuum; the average friction force in the vicinity of the vacancy (estimated from the work required for bringing the tip from $R_{x} / l_{a}=0$ to 1 at $R_{z}=3.92 \AA$ in Fig. 3 ) is approximately $400 \times 10^{-12} \mathrm{~N}$ in the liquid while it is $1 \times 10^{-12} \mathrm{~N}$ in vacuum.

At the constant-load mode, the stable trajectory of the tip defined as a series of the most probable heights is either discontinuous or continuous depending on the magnitude of the load. In particular, it is found that under a certain load

*Present address: Department of Chemistry, Fukuoka University of Education, Munakata, Fukuoka 811-4192, Japan.

${ }^{1}$ G. Binnig, C. F. Quate, and Ch. Gerber, Phys. Rev. Lett. 56, 930 (1986).

${ }^{2}$ L. D. Gelb and R. M. Lynden-Bell, Chem. Phys. Lett. 211, 328 (1993).

${ }^{3}$ L. D. Gelb and R. M. Lynden-Bell, Phys. Rev. B 49, 2058 (1994).

${ }^{4}$ D. L. Patrick and R. M. Lynden-Bell, Surf. Sci. 380, 224 (1997).

${ }^{5}$ K. Koga and X. C. Zeng, Phys. Rev. Lett. 79, 853 (1997).

${ }^{6}$ S. J. Oshea, M. E. Welland, and T. Rayment, Appl. Phys. Lett. 60, 2356 (1992).

${ }^{7}$ J. P. Cleveland, T. E. Schäffer, and P. K. Hansma, Phys. Rev. B 52, R8692 (1995).

${ }^{8}$ W. Han and S. M. Lindsay, Appl. Phys. Lett. 72, 1656 (1998).

${ }^{9}$ V. Koutsos, E. Manias, G. ten Brinke, and G. Hadziioannou, Europhys. Lett. 26, 103 (1994).

${ }^{10}$ S. W. Chen and P. J. Rossky, J. Phys. Chem. 97, 6078 (1993).

${ }^{11}$ M. Matsumoto, H. Tanaka, and K. Nakanishi, J. Chem. Phys. 99, 6935 (1993).

${ }^{12}$ D. Chandler and H. C. Andersen, J. Chem. Phys. 57, 1930 (1972).

${ }^{13}$ K. Koga, X. C. Zeng, and H. Tanaka, J. Chem. Phys. 106, 9781 (around $60 \mathrm{pN}$ per atom of the tip in this model) the trajectory shows no discontinuity in the vicinity of the vacancy but periodic discontinuity elsewhere.

Finally, we would like to point out that compared to typical tip size of laboratory AFM $(\sim 50 \mathrm{~nm}$ in diameter $)$ the model tip considered in this study is too small $(\sim 1 \mathrm{~nm}$ in diameter). It has been proposed that future generation AFM tip, e.g., using carbon nanotube, ${ }^{23}$ will reach nanometer size in diameter. By then a direct comparison with AFM experiments in liquid environment will be feasible.

\section{ACKNOWLEDGMENTS}

This work was supported by JSPS (K.K.) and by NSF and ONR (X.C.Z).

(1997).

${ }^{14}$ F. Hirata and R. M. Levy, Chem. Phys. Lett. 136, 267 (1987); K. S. Schweizer and J. G. Curro, Phys. Rev. Lett. 58, 246 (1987).

${ }^{15}$ N. Sasaki and M. Tsukada, Phys. Rev. B 52, 8471 (1995); N. Sasaki, K. Kobayashi, and M. Tsukada, ibid. 54, 2138 (1996).

${ }^{16}$ W. B. Sediawan, S. Gupta, and E. McLaughlin, J. Chem. Phys. 90, 1888 (1989).

${ }^{17}$ W. Jorgensen, J. Am. Chem. Soc. 103, 335 (1981); B. M. Pettitt and P. J. Rossky, J. Chem. Phys. 77, 1451 (1982).

${ }^{18}$ For example, see A. Ben-Naim, in Solvation Thermodynamics (Plenum Press, New York, 1987), p. 123.

${ }^{19}$ F. Hirata, B. M. Pettitt, and P. J. Rossky, J. Chem. Phys. 77, 509 (1982).

${ }^{20}$ S. J. Singer and D. Chandler, Mol. Phys. 55, 621 (1985).

${ }^{21}$ D. A. Zichi and P. J. Rossky, J. Chem. Phys. 84, 1712 (1986).

${ }^{22}$ J. P. Hansen and I. R. McDonald, Theory of Simple Liquids (Academic, London, 1990), p. 485.

${ }^{23}$ H. Dai, J. H. Hafner, A. G. Rinzler, D. T. Colbert, and R. E. Smalley, Nature (London) 384, 147 (1996); D. J. Keller, ibid. 384, 111 (1996). 